\section{SRL RECORD COPY}

\section{5}



DPST-89-281

Keywords: Waste Analyses,

Waste Tank Certification

Retention Period: Permanent

H. H. Rowland, 703-F

H. D. Harmon, 773-A

C. E. Coffey, 773-A

W. A. Spencer, 773-A

C. W. Jenkins, 773-A

SRL Records (4) 773-A

March 28, 1989

To: $\quad$ K. Andringa, 773-41A

From: C. J. Coleman, 773-A

\author{
ceClomex
}

\title{
ANALYSIS OF SRP WASTE STREAMS FOR WASTE TANK CERTIFICATION (U) \\ SUMMARY
}

SRP waste streams were analyzed to enable SRP to apply for a permit to operate the High-Level Waste Tanks. This report transfers the analytical results from SRL to the Waste Management Technology Department and discusses the analytical methods that were used.

SRP is preparing a RCRA Part B permit application to operate the F and H-Area Tank Farm. The permit is needed to comply with the South Carolina Hazardous Waste Management Regulation R.6179.270.14(b), October 28, 1988. This regulation requires characterization of hazardous wastes and inclusion of this information in the permit application.

The Analytical Development Division (ADD) characterized the waste streams by determining 21 components in each stream. Analyses were performed on the following streams:

- F-Area Low-Heat Waste

- F-Area High-Heat Waste

- F-Area Evaporator Overheads Waste

- H-Area Low-Heat Waste

- H-Area High-Heat Waste

- H-Area Waste Management Maintenance Facility Waste

- Receiving Basin for Offsite Fuel/Resin Regeneration Facility Waste

- K-Area Filter Backwash Waste

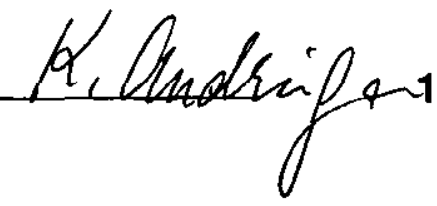




\section{INTRODUCTION}

SRP will apply for certification from the State of South Carolina to operate the SRP High-Level Waste Tanks. The permit application will be submitted as a RCRA Part B, Volume 16, entitled "RCRA Part B Application For the F and H-Area Radioactive Waste Farm".

RCRA regulations require that influent and effluent streams of hazardous waste sites be characterized to obtain an operating permit. The Waste Management Technology Department requested ADD to determine 21 components (including $\mathrm{pH}$ and weight percent solids) in the current influent streams to SRP High-Level Waste Tanks. The analyses will be used to supplement existing data on the composition of High-Level Waste. Effluent streams, which will feed Saltstone and the DWPF, will be analyzed when they are produced.

This report contains the data obtained from analyzing key influent streams to SRP High-Level Waste Tanks. The precision of the data and the analytical methods that were used are also discussed.

\section{DISCUSSION}

\section{SPECIFICATION OF COMPONENTS TO BE DETERMINED}

P. D. d'Entremont of the Waste Management Technology Department specified the set of determinations to be made on waste streams. The following criteria were used in selecting the determinations:

1. Metals listed in the EPA toxicity test that have been found in SRP waste (Ba, $\mathrm{Cr}, \mathrm{Pb}, \mathrm{Hg}, \mathrm{Ag}$ ).

2. Elements that have been shown to have significant concentrations in SRP waste ( $\mathrm{Na}, \mathrm{Mn}, \mathrm{Al}, \mathrm{Fe}, \mathrm{P}, \mathrm{Si}, \mathrm{Ti}, \mathrm{K})$.

Reference:

R. E. Eibling and J. R. Fowler, "Updated Waste Composition At The Savannah River Plant", DPST-88-313.

3. Other components needed to characterize the waste $(\mathrm{pH}$, weight percent solids, anions, total carbon, total and free hydroxide).

\section{EXPERIMENTAL-GENERAL}

Several waste streams were represented by combining samples from individual feed tanks in the correct volume ratios 1 . Other streams needed sampling from only one tank.

Samples containing insoluble solids were dissolved prior to elemental analysis. The slurries were dried for 16 hours at 115 degrees Centigrade to yield a powder. About $0.2 \mathrm{~g}$ of powder was combined with $12 \mathrm{~mL}$ of aqua regia ( 3 parts concentrated $\mathrm{HCl}$ to 1 part concentrated $\mathrm{HNO}_{3}$ ) in a Teflon dissolution container. The container was sealed and placed in an oven set at 115 degrees Centigrade for 2 hours. After cooling, the vessel was opened and the contents poured into a tared $125 \mathrm{~mL}$ plastic bottle. Deionized, distilled water was added until the final solution weight was about $100 \mathrm{~g}$. 
Dilutions performed in shielded cells were made by weighing an aliquot of the sample and then adding deionized, distilled water to obtain the desired dilution.

\section{EXPERIMENTAL-SAMPLE PREPARATION}

\section{F-AREA LOW-HEAT WASTE ${ }^{2}$}

Samples from four waste tanks were combined in the following percentages:

$\begin{array}{llr}\text { Tank } & 5.2 & -27 \% \\ \text { Tank } 6.8 & -26 \% \\ \text { Tank } 18.7- & 6 \% \\ \text { Tank } 710-41 \%\end{array}$

The composite slurry was analyzed directly for weight percent solids and $\mathrm{pH}$. The remaining slurry was then divided into two portions for treatment prior to subsequent analyses. One portion was filtered and the filtrate analyzed for anions, hydroxide, and total carbon. The other portion was dried and dissolved with hot aqua regia for elemental analysis. The determinations for this stream, performed in triplicate, are compiled in Table 1.

\section{F-AREA HIGH-HEAT WASTE 3}

One peanut vial $(12 \mathrm{~mL})$ of Tank 12.1 slurry was available for analysis. The slurry was analyzed directly for weight percent solids and $\mathrm{pH}$. The remaining slurry was divided into two portions for filtration and dissolution for subsequent analyses. The filtrate was diluted by a factor of $9.07(2.774$ $\mathrm{g}$ to $25.158 \mathrm{~g}$ ) to obtain enough sample for anion, hydroxide, and total carbon determinations. The other portion was dried and dissolved with hot aqua regia for elemental analysis. the results are compiled in Table 2.

\section{F-AREA OVERHEAD EVAPORATOR ${ }^{4}$}

This liquid sample was analyzed directly without any sample preparation. Table 3 lists the results of the triplicate set of determinations.

\section{H-AREA LOW-HEAT WASTE 5}

H-Area Low-Heat Waste was represented by combining waste from Tank 710 and Tank 8.6 in 1.5 to 1 ratio. Since $10 \mathrm{~mL}$ of Tank 8.6 sample was provided, the total sample volume available for analysis was $25 \mathrm{~mL}$. The slurry sample was analyzed directly for weight percent solids and $\mathrm{pH}$. The remaining sample was divided into two portions. One portion was filtered to yield a filtrate that was used for anion, hydroxide, and carbon determinations. The other portion was dried and dissolved for elemental analysis. Table 4 lists the analytical results of one set of determinations.

\section{H-AREA HIGH-HEAT WASTE 6}

Tank 8.4 sample was diluted to reduce the radiation field from $5 \mathrm{Rad} / \mathrm{hour}$ to a level permissable for hood work. The sample was then analyzed without further preparation. Table 5 lists the analytical results of one set of determinations. 


\section{H-AREA WASTE MANAGEMENT MAINTENANCE FACILITY WASTE ${ }^{7}$}

Tank 299-H sample contained only a trace of insoluble solids. The sample was analyzed directly for $\mathrm{pH}$ and weight percent solids. A portion of the sample was then filtered to yield a clear solution for anion, carbon and hydroxide measurements. To dissolve the solids for metal analysis, a $10 \mathrm{~mL}$ aliquot of the unfiltered sample was heated with $5 \mathrm{~mL}$ of aqua regia for 2 hours at 115 degrees Centigrade. Elemental determinations were performed on the solutions. The results are compiled in Table 6.

\section{RECEIVING BASIN FOR OFFSITE FUEL/RESIN REGENERATION FACILITY WASTE (RBOF/RRFW) ${ }^{8}$}

Samples from five tanks were mixed in the following percentages to produce a sample representative of RBOF/RRF Waste:

Tritium Target Cleaning

$49 \%$

RBOF Liquid Waste

$33 \%$

Resin Regeneration

$6 \%$

Floating Out Resin

Resin Regeneration

$6 \%$

Regeneration Waste

$6 \%$

Resin Regeneration

Floating Resin Back Into

The Deionizer

Since the composite sample contained no solids, it was analyzed directly without further preparation. The analytical results of triplicate determinations are compiled in Table 7.

\section{K-AREA FILTER BACKWASH ${ }^{9}$}

This sample was divided into two portions for separate treatment. One portion was analyzed directly for $\mathrm{pH}$ and weight percent solids. This portion was then filtered to yield a filtrate for anion, hydroxide, and carbon analyses. The other portion was dried and dissolved for elemental analysis. The analytical results of triplicate determinations are compiled in Table 8.

\section{UNITS OF THE DETERMINATIONS}

All determinations are reported in units of milligrams per liter $(\mathrm{mg} / \mathrm{L})$ except for weight percent solids, which are reported in percent, and $\mathrm{pH}$, which is unitless. It should be noted that the determinations of slurry samples are all reported on a slurry basis, even though elemental determinations required that the slurry be dried and then dissolved. The weight percent solids in the original sample was used to convert dry basis determinations to a slurry basis. 


\section{ANALYTICAL METHODS, DETECTION LIMITS, AND PRECISION OF DETERMINATIONS}

Table 9 lists the analytical method and the estimated detection limit for each analyte. The theoretical detection limits in the first column are those that can be obtained under ideal conditions (i.e., instrument calibration conditions). The detection limits of analytes are increased if the sample must be diluted prior to analysis. Dilutions were required either to reduce radioactivity or to dissolve the solids in slurry samples. The increase in detection limits above the theoretical limit reflects the dilution factor used for each analyte in the waste stream.

The control limits of methods as stated in the monthly ADD QA report ${ }^{10}$ are listed in Table 10 . It is useful to compare these control limits with the observed precision (percent RSD) of the waste streamdeterminations as shown in Tables $1,3,6,7$, and 8. The precision of waste stream determinations, as expected, are generally poorer than QA control limits. The concentration of many of the analytes is near or below the method detection limit. To achieve optimum precision, the analyte concentration should be at least 10 times the detection limit. Moreover, the precision values were based on only three determinations, which is too few to obtain optimum precision statistics.

\section{QUALITY ASSURANCE}

The waste stream analyses were performed with a quality assurance plan in place. Elemental, anion, and hydroxide determinations were performed after method standardizations required to comply with the ADD quality assurance program. Weight percent solids and $\mathrm{pH}$ determinations were made after methods were standardized in the High Level Caves. 


\section{REFERENCES}

1. P. D. d'Entremont, Memorandum to C. J. Coleman, "Mixing Recipes for Tank Farm RCRA Part B Samples", May 18, 1988.

2. C. J. Coleman, Technical Notebook, DPSTN-4265, pp. 107-108.

3. C. J. Coleman, Technical Notebook, DPSTN-4265, pp. 120-121.

4. C. J. Coleman, Technical Notebook, DPSTN-4265, pp. 128-129.

5. C. J. Coleman, Technical Notebook, DPSTN-4265, PP. 116-117.

6. C. J. Coleman, Technical Notebook, DPSTN-4265, pp. 118-119.

7. C. J. Coleman, Technical Notebook, DPSTN-4265, pp. 130-131

8. C. J. Coleman, Technical Notebook, DPSTN-4265, pp. 114-115.

9. C. J. Coleman, Technical Notebook, DPSTN-4265, pp. 126-127.

10. H. B. Aiken, DPSTQA-89-177-3. 
TABLE 1

DETERMINATION OF F-AREA

LOW-HEAT WASTE

DETERMINATION

Total Wt. \% Solids

$\mathrm{pH}$

Free Hydroxide

Total Hydroxide

Total Carbon

Nitrate

Nitrite

Sulfate

$\mathrm{Cr}$

$\mathrm{Ba}$

$\mathrm{Pb}$

$\mathrm{Ag}$

$\mathrm{Na}$

$\mathrm{Mn}$

Al

$\mathrm{Fe}$

$\mathbf{P}$

Si

$\mathrm{Ti}$

K

$\mathrm{Hg}$

UNITS VALUE $\frac{\text { VALUE }}{\text { SET } 2}$

wt $\%$

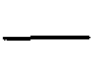

$\mathrm{mg} / \mathrm{L}$

$\mathrm{mg} / \mathrm{L}$

$\mathrm{mg} / \mathrm{L}$

$\mathrm{mg} / \mathrm{L}$

$\mathrm{mg} / \mathrm{L}$

$\mathrm{mg} / \mathrm{L}$

$\mathrm{mg} / \mathrm{L}$

$\mathrm{mg} / \mathrm{L}$

$\mathrm{mg} / \mathrm{L}$

$\mathrm{mg} / \mathrm{L}$

$\mathrm{mg} / \mathrm{L}$

$\mathrm{mg} / \mathrm{L}$

$\mathrm{mg} / \mathrm{L}$

$\mathrm{mg} / \mathrm{L}$

$\mathrm{mg} / \mathrm{L}$

$\mathrm{mg} / \mathrm{L}$

$\mathrm{mg} / \mathrm{L}$

$\mathrm{mg} / \mathrm{L}$

$\mathrm{mg} / \mathrm{L}$
29.27

13.01

24,100

26,900

319

220,000

3,600

6,350

90

60

30

$<30$

86,200

30

5,500

1,900

700

700

$<3$

3

3
29.19

12.90

24,500

26,900

388

218,000

3,600

6,225

60

30

30

$<30$

86,000

$<30$

6,200

900

400

300

$<3$

3

3
YALUE

SET 3

29.55

13.04

24,500

27,000



217,000

3,550

6,200

60

30

30

$<30$

87,200

$<30$

6,100

1,500

200

600

$<3$

3

3
AVERAGE

29.34

12.98

24,500

26,966

354

218,000

3,570

6,250

70

40

30

$<30$

86,500

$0.7 \%$

$<30$

5,900

$6.4 \%$

$35 \%$

$58 \%$

$39 \%$

533

$<3$

3

3

$0.6 \%$

$0.6 \%$

$0.9 \%$

$0.2 \%$

$0.7 \%$

$0.5 \%$

$1.3 \%$

$24 \%$

$43 \%$

$0 \%$

$1,400 \quad 35 \%$

433

533

$0 \%$

\footnotetext{
* $\%$ RSD $=\%$ RELATIVE STANDARD DEVIATION
} 
TABLE 2

DETERMINATION OF F-AREA

HIGH-HEAT WASTE

\begin{tabular}{|c|c|c|}
\hline DETERMINATLON & UNITS & YALUE \\
\hline Total Wt. \% Solids & $w t \%$ & 36.1 \\
\hline $\mathrm{pH}$ & - & 12.8 \\
\hline Free Hydroxide & $\mathrm{mg} / \mathrm{L}$ & 4,760 \\
\hline Total Hydroxide & $\mathrm{mg} / \mathrm{L}$ & 14,000 \\
\hline Total Carbon & $\mathrm{mg} / \mathrm{L}$ & Insufficient sample to determine \\
\hline Nitrate & $\mathrm{mg} / \mathrm{L}$ & 181,000 \\
\hline Nitrite & $\mathrm{mg} / \mathrm{L}$ & 9,600 \\
\hline $\mathrm{Cr}$ & $\mathrm{mg} / \mathrm{L}$ & 200 \\
\hline $\mathrm{Ba}$ & $\mathrm{mg} / \mathrm{L}$ & $<30$ \\
\hline $\mathrm{Pb}$ & $\mathrm{mg} / \mathrm{L}$ & 30 \\
\hline $\mathrm{Ag}$ & $\mathrm{mg} / \mathrm{L}$ & $<30$ \\
\hline $\mathrm{Na}$ & $\mathrm{mg} / \mathrm{L}$ & 91,000 \\
\hline $\mathrm{Mn}$ & $\mathrm{mg} / \mathrm{L}$ & 40 \\
\hline $\mathrm{Al}$ & $\mathrm{mg} / \mathrm{L}$ & 2,800 \\
\hline $\mathrm{Fe}$ & $\mathrm{mg} / \mathrm{L}$ & 6,100 \\
\hline $\mathbf{P}$ & $\mathrm{mg} / \mathrm{L}$ & 400 \\
\hline $\mathrm{Si}$ & $\mathrm{mg} / \mathrm{L}$ & 60 \\
\hline $\mathrm{Ti}$ & $\mathrm{mg} / \mathrm{L}$ & $<10$ \\
\hline $\mathbf{K}$ & $\mathrm{mg} / \mathrm{L}$ & $<140$ \\
\hline $\mathrm{Hg}$ & $\mathrm{mg} / \mathrm{L}$ & 1100 \\
\hline
\end{tabular}


TABLE 3

DETERMINATION OF F-AREA

OVERHEAD EVAPORATOR WASTE

\begin{tabular}{|c|c|c|c|c|c|c|}
\hline DETERMUNATION & UNUTS & $\frac{\text { VALUE }}{\text { SET } 1}$ & $\frac{\text { VALUE }}{\text { SET } 2}$ & $\frac{\text { VALUE }}{\text { SET } 3}$ & AVERAGE & \% $\mathrm{RSD}^{*}$ \\
\hline Total Wt. \% Solids & wt $\%$ & 1.43 & 1.24 & 1.30 & 1.32 & $7.3 \%$ \\
\hline $\mathrm{pH}$ & $\longrightarrow$ & 9.5 & $\longrightarrow$ & $\longrightarrow$ & & - \\
\hline Free Hydroxide & $\mathrm{mg} / \mathrm{L}$ & $<10$ & $<10$ & $<10$ & $<10$ & - \\
\hline Total Hydroxide & $\mathrm{mg} / \mathrm{L}$ & $<50$ & $<50$ & $<50$ & $<50$ & - \\
\hline Total Carbon & $\mathrm{mg} / \mathrm{L}$ & 22.5 & 20.9 & 22.5 & 22.0 & $4.2 \%$ \\
\hline Nitrate & $\mathrm{mg} / \mathrm{L}$ & $<1.5$ & $<1.5$ & $<1.5$ & $<1.5$ & \\
\hline Nitrite & $\mathrm{mg} / \mathrm{L}$ & $<1$ & 1.15 & 1.16 & -1 & - \\
\hline Sulfate & $\mathrm{mg} / \mathrm{L}$ & $<0.5$ & $<0.5$ & $<0.5$ & $<0.5$ & \\
\hline $\mathrm{Cr}$ & $\mathrm{mg} / \mathrm{L}$ & $<0.005$ & $<0.005$ & $<0.005$ & $<0.005$ & \\
\hline $\mathrm{Ba}$ & $\mathrm{mg} / \mathrm{L}$ & $<0.005$ & $<0.005$ & $<0.005$ & $<0.005$ & \\
\hline $\mathrm{Pb}$ & $\mathrm{mg} / \mathrm{L}$ & $<0.02$ & $<0.02$ & $<0.02$ & $<0.02$ & \\
\hline $\mathrm{Ag}$ & $\mathrm{mg} / \mathrm{L}$ & $<0.01$ & $<0.01$ & $<0.01$ & $<0.01$ & $\ldots$ \\
\hline $\mathrm{Na}$ & $\mathrm{mg} / \mathrm{L}$ & 0.52 & 0.51 & 0.51 & 0.51 & $2.0 \%$ \\
\hline $\mathrm{Mn}$ & $\mathrm{mg} / \mathrm{L}$ & $<0.005$ & $<0.005$ & $<0.005$ & $<0.005$ & $\ldots$ \\
\hline $\mathrm{Al}$ & $\mathrm{mg} / \mathrm{L}$ & $<0.01$ & $<0.01$ & $<0.01$ & $<0.01$ &  \\
\hline $\mathrm{Fe}$ & $\mathrm{mg} / \mathrm{L}$ & $<0.005$ & $<0.005$ & $<0.005$ & $<0.005$ & - \\
\hline$P$ & $\mathrm{mg} / \mathrm{L}$ & 0.02 & 0.01 & 0.01 & 0.01 & $58 \%$ \\
\hline $\mathrm{Si}$ & $\mathrm{mg} / \mathrm{L}$ & 2.69 & 2.68 & 2.69 & 2.69 & $0.2 \%$ \\
\hline $\mathrm{Ti}$ & $\mathrm{mg} / \mathrm{L}$ & $<0.005$ & $<0.005$ & $<0.005$ & $<0.005$ & \\
\hline $\mathbf{K}$ & $\mathrm{mg} / \mathrm{L}$ & $<0.014$ & $<0.014$ & $<0.014$ & $<0.014$ & {[} \\
\hline $\mathrm{Hg}$ & $\mathrm{mg} / \mathrm{L}$ & 0.003 & 0.002 & 0.020 & 0.008 & $122 \%$ \\
\hline
\end{tabular}

* $\%$ RSD $=\%$ RELATIVE STANDARD DEVIATION 
TABLE 4

DETERMINATION OF H-AREA

LOW-HEAT WASTE

\begin{tabular}{|c|c|c|}
\hline DETERMINATLON & UNUTS & YALUE \\
\hline Total Wt. \% Solids & wt $\%$ & 30.00 \\
\hline $\mathrm{pH}$ & $\longrightarrow$ & 11.7 \\
\hline Free Hydroxide & $\mathrm{mg} / \mathrm{L}$. & 119 \\
\hline Total Hydroxide & $\mathrm{mg} / \mathrm{L}$ & 2,500 \\
\hline Total Carbon & $\mathrm{mg} / \mathrm{L}$ & 2.0 \\
\hline Nitrate & $\mathrm{mg} / \mathrm{L}$ & 234,000 \\
\hline Nitrite & $\mathrm{mg} / \mathrm{L}$ & $<500$ \\
\hline Sulfate & $\mathrm{mg} / \mathrm{L}$ & 9,500 \\
\hline $\mathrm{Cr}$ & $\mathrm{mg} / \mathrm{L}$ & 90 \\
\hline $\mathrm{Ba}$ & $\mathrm{mg} / \mathrm{L}$ & $<10$ \\
\hline $\mathrm{Pb}$ & $\mathrm{mg} / \mathrm{L}$ & 30 \\
\hline Ag & $\mathrm{mg} / \mathrm{L}$ & $<10$ \\
\hline $\mathrm{Na}$ & $\mathrm{mg} / \mathrm{L}$ & 80,700 \\
\hline $\mathrm{Mn}$ & $\mathrm{mg} / \mathrm{L}$ & 10 \\
\hline $\mathrm{Al}$ & $\mathrm{mg} / \mathrm{L}$ & 200 \\
\hline $\mathrm{Fe}$ & $\mathrm{mg} / \mathrm{L}$ & 2,600 \\
\hline $\mathbf{P}$ & $\mathrm{mg} / \mathrm{L}$ & 500 \\
\hline Si & $\mathrm{mg} / \mathrm{L}$ & 10 \\
\hline $\mathrm{Ti}$ & $\mathrm{mg} / \mathrm{L}$ & $<10$ \\
\hline $\mathbf{K}$ & $\mathrm{mg} / \mathrm{L}$ & 200 \\
\hline $\mathrm{Hg}$ & $\mathrm{mg} / \mathrm{L}$ & 10 \\
\hline
\end{tabular}


TABLE 5

DETERMINATION OF H.AREA

HIGH-HEAT WASTE

\begin{tabular}{|c|c|c|}
\hline DETERMUNATION & UNITS & VALUE \\
\hline Total Wt. \% Solids & wt $\%$ & 19.9 \\
\hline $\mathrm{pH}$ & $\underline{-}$ & 0.0 \\
\hline Free Hydroxide & $\mathrm{mg} / \mathrm{L}$ & $<1$ \\
\hline Total Hydroxide & $\mathrm{mg} / \mathrm{L}$ & $<1$ \\
\hline Total Carbon & $\mathrm{mg} / \mathrm{L}$ & 1,620 \\
\hline Nitrate & $\mathrm{mg} / \mathrm{L}$ & 260,000 \\
\hline Nitrite & $\mathrm{mg} / \mathrm{L}$ & $<25$ \\
\hline Sulfate & $\mathrm{mg} / \mathrm{L}$ & 4,400 \\
\hline $\mathrm{Cr}$ & $\mathrm{mg} / \mathrm{L}$ & 33.2 \\
\hline $\mathrm{Ba}$ & $\mathrm{mg} / \mathrm{L}$ & 19.0 \\
\hline $\mathrm{Pb}$ & $\mathrm{mg} / \mathrm{L}$ & 23.7 \\
\hline $\mathrm{Ag}$ & $\mathrm{mg} / \mathrm{L}$ & $<5.0$ \\
\hline $\mathrm{Na}$ & $\mathrm{mg} / \mathrm{L}$ & 5,300 \\
\hline Mn & $\mathrm{mg} / \mathrm{L}$ & 475 \\
\hline $\mathrm{Al}$ & $\mathrm{mg} / \mathrm{L}$ & 18,600 \\
\hline $\mathrm{Fe}$ & $\mathrm{mg} / \mathrm{L}$ & 1,330 \\
\hline $\mathbf{P}$ & $\mathrm{mg} / \mathrm{L}$ & 85.5 \\
\hline $\mathrm{Si}$ & $\mathrm{mg} / \mathrm{L}$ & $<5.0$ \\
\hline $\mathrm{Ti}$ & $\mathrm{mg} / \mathrm{L}$ & $<2.4$ \\
\hline $\mathrm{K}$ & $\mathrm{mg} / \mathrm{L}$ & 200 \\
\hline $\mathrm{Hg}$ & $\mathrm{mg} / \mathrm{L}$ & 356 \\
\hline
\end{tabular}


TABLE 6

DETERMINATION OF WASTE MANAGEMENT

MAINTENANCE FACILITY WASTE

\begin{tabular}{|c|c|c|c|c|c|c|}
\hline DETERMINATION & UNUTS & $\frac{\text { VALUE }}{\text { SET } 1}$ & $\frac{\text { YALUE }}{\text { SET } 2}$ & $\frac{\text { VALUE }}{\text { SET } 3}$ & AVERAGE & \% RSD \\
\hline Total Wt. \% Solids & wt \% & 8.13 & 8.25 & 8.38 & 8.25 & $1.5 \%$ \\
\hline pH & $\ldots$ & 12.66 & 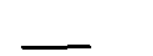 & $\ldots$ & 12.66 & \\
\hline Total Hydroxide & $\mathrm{mg} / \mathrm{L}$ & 1.48 & 1.48 & 1.46 & 1.47 & $0.80 \%$ \\
\hline Free Hydroxide & $\mathrm{mg} / \mathrm{L}$ & 1.45 & 1.45 & 1.43 & 1.44 & $0.80 \%$ \\
\hline Total Carbon & $\mathrm{mg} / \mathrm{L}$ & 1130 & 1100 & 1190 & 1140 & $4.0 \%$ \\
\hline Nitrate & $\mathrm{mg} / \mathrm{L}$ & 6070 & 6310 & 6100 & 6160 & $2.1 \%$ \\
\hline Nitrite & $\mathrm{mg} / \mathrm{L}$ & $<25$ & $<25$ & $<25$ & $<25$ & $\underline{-}$ \\
\hline Sulfate & $\mathrm{mg} / \mathrm{L}$ & 12.5 & 12.5 & 12.5 & 12.5 & - \\
\hline $\mathrm{Cr}$ & $\mathrm{mg} / \mathrm{L}$ & 16.3 & 16.3 & 18.8 & 17.0 & $7.5 \%$ \\
\hline $\mathrm{Ba}$ & $\mathrm{mg} / \mathrm{L}$ & 0.10 & 0.10 & 0.08 & 0.09 & $12.4 \%$ \\
\hline $\mathrm{Pb}$ & $\mathrm{mg} / \mathrm{L}$ & 25.0 & 22.8 & 29.4 & 25.7 & $13.2 \%$ \\
\hline $\mathrm{Ag}$ & $\mathrm{mg} / \mathrm{L}$ & $<0.01$ & $<0.01$ & $<0.01$ & $<0.01$ & $\underline{-}$ \\
\hline $\mathrm{Na}$ & $\mathrm{mg} / \mathrm{L}$ & 39,770 & 34,400 & 34,000 & 36,000 & $8.9 \%$ \\
\hline $\mathrm{Mn}$ & $\mathrm{mg} / \mathrm{L}$ & 4.32 & 5.04 & 4.52 & 4.62 & $8.0 \%$ \\
\hline $\mathrm{Al}$ & $\mathrm{mg} / \mathrm{L}$ & 7.33 & 7.44 & 7.28 & 7.35 & $1.1 \%$ \\
\hline $\mathrm{Fe}$ & $\mathrm{mg} / \mathrm{L}$ & 393 & 369 & 365 & 376 & $4.0 \%$ \\
\hline $\mathbf{P}$ & $\mathrm{mg} / \mathrm{L}$ & 32.0 & 34.7 & 34.2 & 33.6 & $4.3 \%$ \\
\hline $\mathrm{Si}$ & $\mathrm{mg} / \mathrm{L}$ & 117 & 115 & 108 & 113 & $4.2 \%$ \\
\hline $\mathrm{Ti}$ & $\mathrm{mg} / \mathrm{L}$ & 1.75 & 1.61 & 1.62 & 1.66 & $4.9 \%$ \\
\hline $\mathrm{K}$ & $\mathrm{mg} / \mathrm{L}$ & 13.08 & 13.48 & 12.81 & 13.12 & $2.6 \%$ \\
\hline $\mathrm{Hg}$ & $\mathrm{mg} / \mathrm{L}$ & 0.92 & 0.75 & 0.85 & 0.84 & $8.5 \%$ \\
\hline
\end{tabular}


TABLE 7

DETERMINATION OF RBOF/RRF WASTE

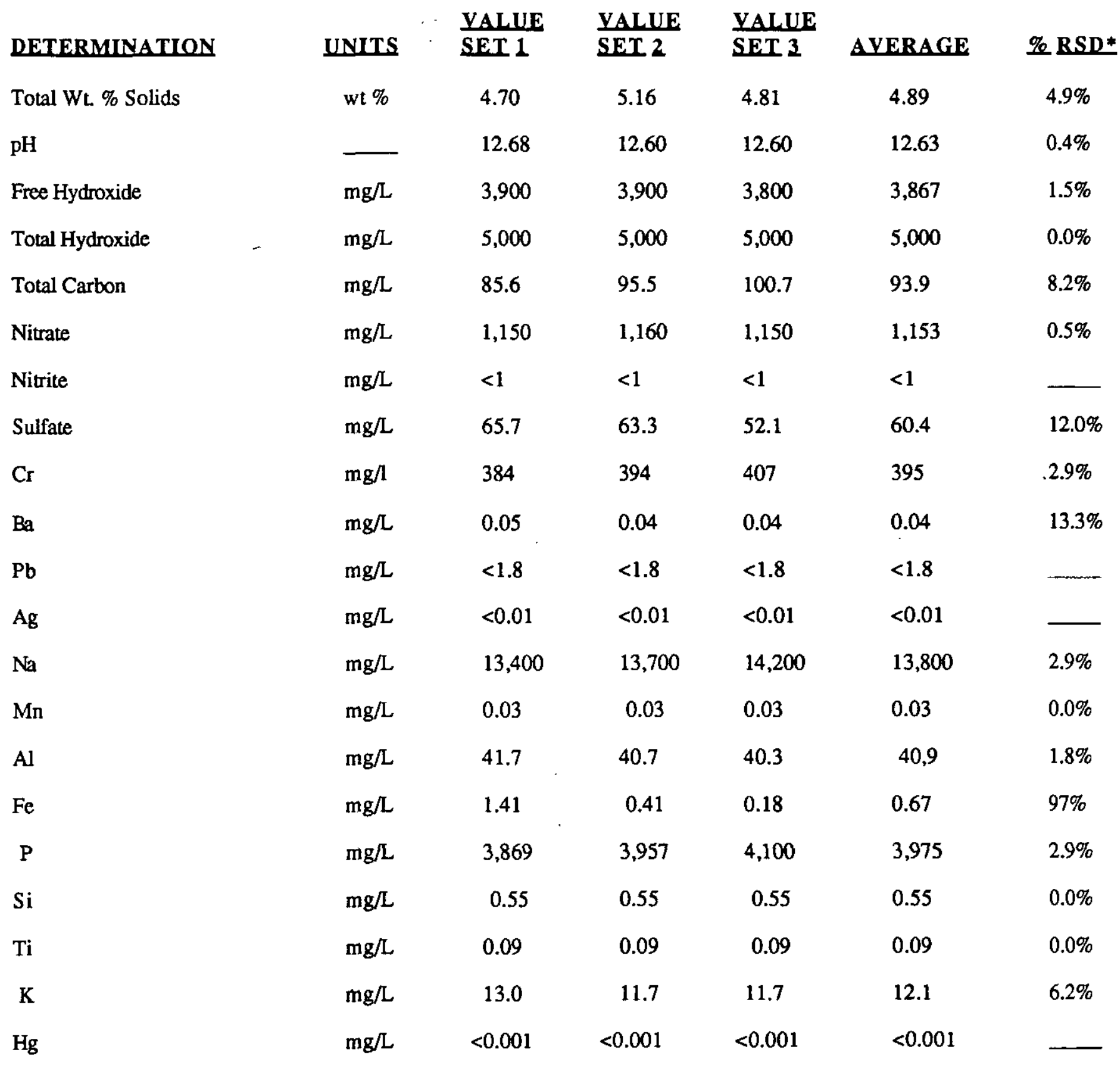

\footnotetext{
* \% RSD $=\%$ RELATIVE STANDARD DEVIATION
} 
TABLE 8

DETERMINATION OF K-AREA

FILTER BACKWASH WASTE

\section{DETERMINATION}

Total Wt. \% Solids

$\mathrm{pH}$

Free Hydroxide

Total Hydroxide

Total Carbon

Nitrate

Nitrite

Sulfate

$\mathrm{Cr}$

$\mathrm{Ba}$

$\mathrm{Pb}$

$\mathrm{Ag}$

$\mathrm{Na}$

Mn

Al

$\mathrm{Fe}$

P

$\mathrm{Si}$

Ti

K

$\mathrm{Hg}$



4.70

4.93

5.01

4.88

$3.3 \%$

7.18

$<0.001$

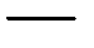

$<0.001$

7.18

$\mathrm{mg} / \mathrm{l}$

$<0.005$

$<0.005$

$<0.001$

$<0.001$

$\mathrm{mg} / \mathrm{L}$

41.6

41.4

$<0.005$

$<0.005$

$\mathrm{mg} / \mathrm{L}$

41.6

41.5

1.95

1.85

$\mathrm{mg} / \mathrm{L}$

1.87

1.74

$<1$

$<1$

$\mathrm{mg} / \mathrm{L}$

$<1$

$<1$

3.39

3.57

4.31

$33 \%$

$\mathrm{mg} / \mathrm{L}$

10

10

10

$0.0 \%$

$\mathrm{mg} / \mathrm{L} \quad 10$

10

10

10

$0.0 \%$

$\mathrm{mg} / \mathrm{L} \quad 30$

30

30

30

$0.0 \%$

$\mathrm{mg} / \mathrm{L}<10$

$<10$

$<10$

$<10$

$\mathrm{mg} / \mathrm{L}$

310

180

183

$68 \%$

$\mathrm{mg} / \mathrm{L} \quad 30$

40

30

33

$17 \%$

$\mathrm{mg} / \mathrm{L} \quad 7,100$

7,300

7,100

7,200

$1.6 \%$

$\mathrm{mg} / \mathrm{L} \quad 4,600$

4,700

4,700

4,666

$1.2 \%$

$\mathrm{mg} / \mathrm{L} \quad 250$

250

250

250

$0.0 \%$

$\mathrm{mg} / \mathrm{L} \quad 20$

80

50

50

$60 \%$

$\mathrm{mg} / \mathrm{L}$

10

10

10

$0.0 \%$

$\mathrm{mg} / \mathrm{L} \quad 20$

20

20

20

$0.0 \%$

$\mathrm{mg} / \mathrm{L}$

$<1$

$<1$

$<1$

$<1$

* $\%$ RSD $=\%$ RELATIVE STANDARD DEVIATION 
TABLE 9

ANALYTICAL METHODS AND DETECTION LIMITS

$\begin{array}{ll}\text { METHOD } & \text { DETERMINATION } \\ \text { Microwave } & \mathrm{Wt} \% \text { Solids } \\ \text { Titration } & \mathrm{Free}^{-} \\ \text {Titration } & \text { Total } \mathrm{OH}^{-} \\ \text {Carbon Analyzer } & \mathrm{Total}^{-} \mathrm{Carbon}^{-} \\ \text {Ion Chromatography } & \mathrm{NO}_{3}^{-} \\ \text {Ion Chromatography } & \mathrm{NO}_{2}^{-} \\ \text {Ion Chromatography } & \mathrm{SO}_{4} \\ \text { ICP AES } & \mathrm{Cr} \\ \text { ICP AES } & \mathrm{Ba} \\ \text { ICP-AES } & \mathrm{Pb} \\ \text { ICP-AES } & \mathrm{Ag} \\ \text { ICP-AES } & \mathrm{Na} \\ \text { ICP-AES } & \mathrm{Mn} \\ \text { ICP-AES } & \mathrm{Al} \\ \text { ICP-AES } & \mathrm{Fe} \\ \text { ICP-AES } & \mathrm{P} \\ \text { ICP-AES } & \mathrm{Si} \\ \text { ICP-AES } & \mathrm{Ti} \\ \text { AAS } & \mathrm{Kg} \\ \text { AAS } & \mathrm{Hg}\end{array}$

1. UNITS ARE IN MG/L EXCEPT FOR WEIGHT \% SOLIDS

\begin{tabular}{cc}
$\begin{array}{c}\text { THEORETCAL } \\
\text { DETECTION LMUT }\end{array}$ & E-ALHW \\
\hline 0.1 & 0.1 \\
50 & 50 \\
10 & 10 \\
1 & 1 \\
1 & 1 \\
1 & 1 \\
1 & 1 \\
0.005 & 2.3 \\
0.005 & 2.3 \\
0.02 & 9.2 \\
0.01 & 4.6 \\
0.02 & 9.2 \\
0.005 & 2.3 \\
0.01 & 4.6 \\
0.005 & 2.3 \\
0.02 & 9.2 \\
0.02 & 9.2 \\
0.005 & 2.3 \\
0.014 & 6.5 \\
0.001 & 0.4
\end{tabular}

$\begin{array}{ccc}\text { E-AHHW }^{2} & \text { E-AOEW }^{2} & \text { H-ALHW } \\ 0.1 & 0.1 & 0.1 \\ 455 & 50 & 5 . \\ 91 & 10 & 10 \\ 9 & 1 & 1 \\ 9 & 1 & 1 \\ 9 & 1 & 1 \\ 9 & 1 & 1 \\ 2.3 & 0.005 & 2.4 \\ 2.3 & 0.005 & 2.4 \\ 9.2 & 0.02 & 9.6 \\ 4.6 & 0.01 & 4.8 \\ 9.2 & 0.02 & 9.6 \\ 2.3 & 0.005 & 2.4 \\ 4.6 & 0.01 & 4.8 \\ 2.3 & 0.005 & 2.4 \\ 9.2 & 0.02 & 9.6 \\ 9.2 & 0.02 & 9.6 \\ 2.3 & 0.005 & 2.4 \\ 6.5 & 0.014 & 6.7 \\ 0.4 & 0.001 & 2.4 \\ \text { F-AOEW (F-AREA OVERHEADS WASTE) } \\ \text { H-ALHW (H-AREA LOW HEAT WASTE) }\end{array}$


TABLE 9 (CONT)

ANALYTICAL METHODS AND DETECTION LIMITS

$\begin{array}{ll}\text { METHOD } & \text { DETERMINATION } \\ \text { Microwave } & \mathrm{Wt} \% \text { Solids } \\ \text { Titration } & \mathrm{Free} \mathrm{OH}^{-} \\ \text {Titration } & \text { Total } \mathrm{OH}^{-} \\ \text {Carbon Analyzer } & \mathrm{Total} \mathrm{Carbon}^{-} \\ \text {Ion Chromatography } & \mathrm{NO}_{3}^{-} \\ \text {Ion Chromatography } & \mathrm{NO}^{-} \\ \text {Ion Chromatography } & \mathrm{SO}_{4} \\ \text { ICP AES } & \mathrm{Cr} \\ \text { ICP AES } & \mathrm{Ba} \\ \text { ICP-AES } & \mathrm{Pb} \\ \text { ICP-AES } & \mathrm{Ag} \\ \text { ICP-AES } & \mathrm{Na} \\ \text { ICP-AES } & \mathrm{Mn} \\ \text { ICP-AES } & \mathrm{Al} \\ \text { ICP-AES } & \mathrm{Fe} \\ \text { ICP-AES } & \mathrm{P} \\ \text { ICP-AES } & \mathrm{Si} \\ \text { ICP-AES } & \mathrm{Ti} \\ \text { AAS } & \mathrm{K} \\ \text { AAS } & \mathrm{Hg} \\ \text { A } & \end{array}$

\begin{tabular}{cc} 
DETECTION LMUT $^{1}$ & H-AHHW \\
\hline 0.1 & 0.1 \\
50 & 23750 \\
10 & 4750 \\
1 & 475 \\
1 & 475 \\
1 & 475 \\
1 & 475 \\
0.005 & 2.4 \\
0.005 & 2.4 \\
0.02 & 9.6 \\
0.01 & 4.8 \\
0.02 & 9.6 \\
0.005 & 2.4 \\
0.01 & 4.8 \\
0.005 & 2.4 \\
0.02 & 9.6 \\
0.02 & 9.6 \\
0.005 & 2.4 \\
0.014 & 6.6 \\
0.001 & 0.5 \\
\hline
\end{tabular}

WMMFW $^{2}$
0.1
50
10
1
1
1
1
0.005
0.005
0.02
0.01
0.02
0.005
0.01
0.005
0.02
0.02
0.005
0.014
0.001

\section{BROF/REFW ${ }^{2}$}

0.1

50

10

1

1

1

1

1

0.005

0.005

0.02

0.01

0.02

0.005

0.01

0.005

0.02

0.02

0.005

0.014

0.001

\section{K-AFBW $^{2}$}

0.1

50

10

1

1

1

1

2.5

2.5

10.

5.0

10.0

2.5

5.0

2.5

10.0

10.0

0.005

0.014

0.001

1. UNITS ARE IN MG/L EXCEPT FOR WEIGHT \% SOLIDS

2. H-AHHW (H-AREA HIGH-HEAT WASTE WMMFW (WASTE MANAGEMENT FACILITY WASTE)

RBOF/RRFW (RECEIVING BASIN FOR OFFSITE FUEL/RESIN REGENERATION FACILITY WASTE)

H-ALHW (H-AERA LOW-HEAT WASTE) 
TABLE 10

\section{ADD CONTROL LIMITS FOR}

SELECTED METHODS

\section{METHOD}

ICP-AES

$\operatorname{AAS}(\mathrm{Hg})$

AAS(K)

$\mathrm{pH}$

Weight \% Solids

Total $\mathrm{OH}^{-}$

Free OH

Carbon

Ion Chromatography

\section{CONTROL LIMIT}

$\pm 5 \%$

$\pm 10 \%$

$\pm 10 \%$

$\pm 5 \%$

$\pm 5 \%$

$\pm 3 \%$

$\pm 5 \%$

$\pm 5 \%$

$\pm 5 \%$ 